\title{
A statistical method for display and segmentation of 3D image data
}

\author{
Bahareh Vafadar, Bing Wu and Phil Bones \\ Computational Imaging Group \\ Dept. Electrical \& Computer Engineering, University of Canterbury, Christchurch, N.Z. \\ phil.bones@canterbury.ac.nz
}

\begin{abstract}
A new method for visualisation and segmentation of vessel structures in 3D magnetic resonance angiography (MRA) images is presented. This method uses a simple statistical model of the information stored along parallel rays within the data set to derive a $2 \mathrm{D}$ projection image. Although similar to the maximum image projection (MIP) method, the new method uses a single parameter to achieve a higher contrast-to-noise ratio at a modest computational cost. The same idea is employed to provide a means of segmenting a 3D data set in order to derive a region of support for the purpose of reconstructing image sequences with high temporal resolution.
\end{abstract}

Index Terms-MR angiography; 3D segmentation; Maximum intensity projection.

\section{INTRODUCTION}

The ability to visualise blood vessels is of great importance for many medical imaging modalities and the term 'angiography' is generally applied. CT and MRI scanners can be used to obtain 3D data sets, which allow the extraction of vascular structures, especially if some form of contrast agent has been injected into the bloodstream. Data originating from magnetic resonance angiography (MRA) exhibits some properties which make the application of some volume visualization techniques like ray casting or iso-surface extraction difficult [1]. MRI data sets, for example, contain a significant amount of noise. The high background signal also produces poor contrast images if a straightforward densitometric (X-ray like) projection is used.

The maximum intensity projection (MIP) algorithm is a simple solution to this problem and has proven to be the most popular rendering algorithm for MRA [2], [3]. Note that the term 'rendering' is here used to represent in a general sense a method to generate a 2D image from a 3D data set. To generate an MIP image, the voxels within the 3D data set with maximum intensity that occur along parallel rays traced from the observer's viewpoint are projected onto the visualization plane. This method of projection is the most frequently employed because it conveys the densitometric information of the original images without the need to tune any parameters and its implementation is relatively simple. The main limitations of MIP are that it cannot adequately depict the spatial relationships of overlapping vessels and large bright structures can occlude other structures along rays from two or more directions.

The MIP algorithm is very simple and provides high signalto-noise ratio (SNR) for large, bright vessels [4], [5]. However, by keeping the maximum background signal along each ray, the algorithm causes the average background intensity in the MIP image to be larger than the background intensity in the original image data and thus small vessels, with low initial SNR, have reduced conspicuity [6], [7]. Additionally, since only the maximum value is recorded, the MIP images provide no information regarding vessel overlap or the depth of the vessels. Fig. 1 shows an MIP for a 3D data set obtained by contrast enhanced MRA of the knee region of a normal healthy volunteer.

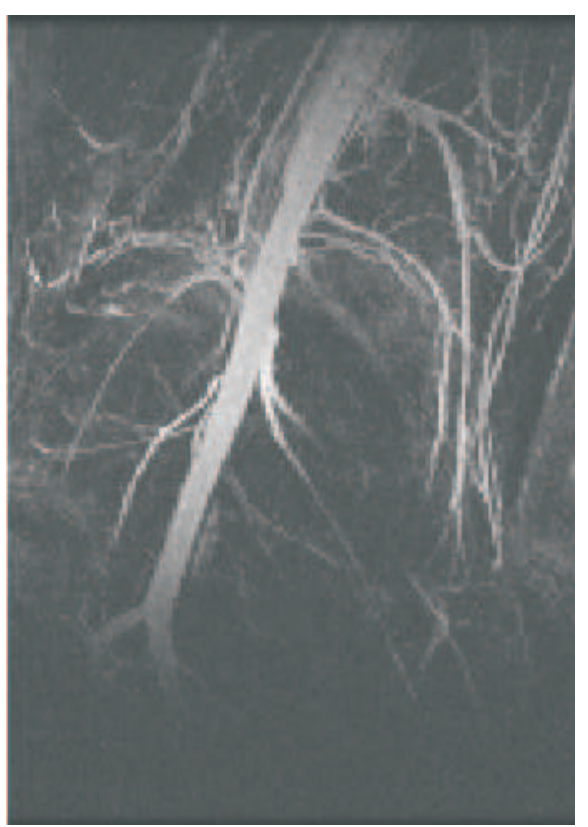

Fig. 1. Coronal MIP image formed from a set of contrast-enhanced MRA data obtained from the knee region of a healthy normal volunteer.

As well as requiring a rendering method for visualising 3D data sets for radiological assessment, we also require a method to segment the $3 \mathrm{D}$ set into those regions which have a high probability of containing significant blood vessels and the rest (the 'background'). We have reported a method for accelerated parallel MRA which relies on the use of a region of support (ROS), i.e. a region outside of which significant changes due to the presence of contrast are unlikely to occur [8], [9], [10]. Knowledge of the ROS allows the elimination of the regions outside the object from the reconstruction, as they are known not to contribute to the signals received in the scanner coils. Comparison between reconstruction methods shows that the 
relative performance of this approach tends to improve with a smaller ROS, provided the ROS still encompasses the vessels of interest. The results have shown that this method is superior to the others in terms of giving lower overall noise levels. In addition, it could be very useful in cases where imaging speed is critical and a small ROS applies. The significance of establishing an ROS has been confirmed by others [11].

In 3D contrast-enhanced MR angiography, the stationary anatomical background can be suppressed via a subtraction to reduce the effective ROS to a large extent. The method we have used to date for definition of that ROS is primitive, however. In brief, three orthogonal MIP images (axial, coronal and sagittal) were segmented by simple global thresholding and application of morphological dilation. The three binary images so formed were backprojected within the 3D volume and combined by a logical AND operation to derive a crude ROS [10].

In the following we present an alternative rendering method to the MIP and demonstrate its use on 3D MRA data sets. We also use the new method to derive an ROS for the purpose of achieving better time resolved MRA image sequences.
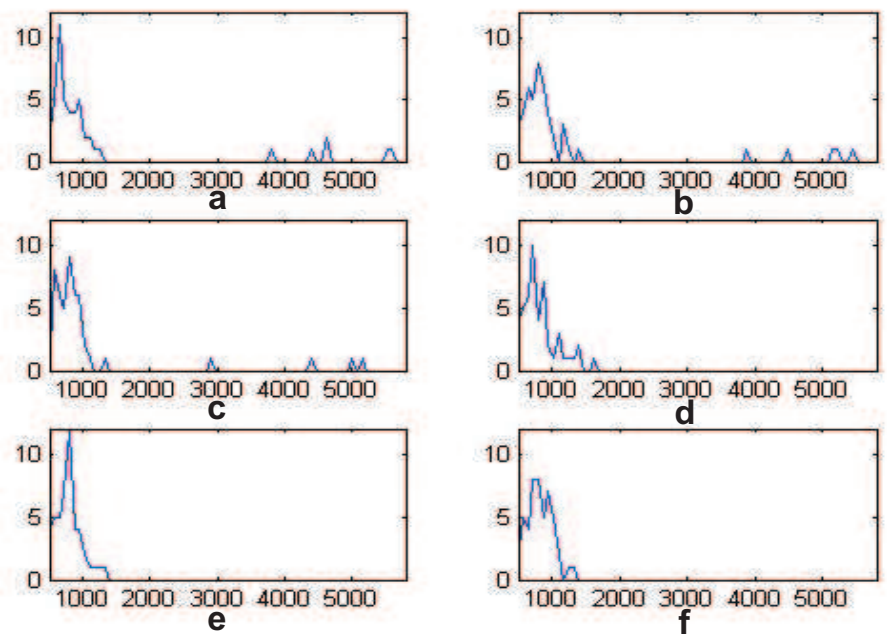

Fig. 2. Histograms of voxel intensities along 6 adjacent parallel rays in the 3D data set shown in Fig. 1. In the first three histograms (a to c), rays intersect the large vessel central in Fig. 1 and thus relatively bright voxels are encountered. The remaining three histograms ( $d$ to $f$ ) are for rays which lie just outside the vessel and appear to comprise background voxels only.

\section{A MODIFIED MIP}

To achieve better visualisation of blood vessels, as well as to enable better 3D segmentation results to be achieved, a method needs to be developed that increases the contrast-tonoise ratio. We propose a modification to MIP which aims to use the maximum value in optical rays which intersect at least one vessel, while selecting the mean value in other cases. In order to achieve this a model is required for the statistical distribution of intensities along rays. We conjecture that virtually all rays comprise a relatively high proportion of non-vessel voxels with intensities belonging to a population with a relatively low intensity (the 'background'). Some rays include as well a relatively small proportion of bright voxels lying inside vessels. The intensity histograms of the latter type of rays therefore contain 'outliers' with respect to the background population.

Fig. 2 illustrates the types of histograms which occur within a typical MRA data set. The background in each case forms a relatively large population of low intensities. When vessels are present they belong to a separate, more intense population. Therefore if a robust measure of the background population can be made, it should be possible to detect the presence of the 'outliers'.

As a robust measure of data location the median is used instead of the mean and as a robust measure of data dispersion the median absolute deviation about the median (MAD) is used [12]. In order to normalise the latter so that it gives a value directly comparable to the standard deviation for a Gaussian distribution, a normalized quantity MADN is defined by

$$
\operatorname{MADN}(x)=\operatorname{MAD}(x) / \operatorname{MAD}(N(0,1)),
$$

where $N\left(\mu, \sigma^{2}\right)$ is a normally distributed random variable with mean $\mu$ and variance $\sigma^{2}$ [12].

As the intensities of voxels lying within vessels are expected to be significantly higher than the background distribution, a threshold for detection can be specified as

$$
T_{\text {ray }}=\text { median }+K \times \mathrm{MADN},
$$

where the median and MADN quantities are estimated for the particular ray concerned and $K$ is a positive scalar. To form a modifed MIP image, then, the threshold is calculated for every ray for a particular viewing direction (usually chosen to be in the direction of one of the cartesian axes). For each pixel in the $2 \mathrm{D}$ projection, if any voxel intensities in the corresponding ray exceed the threshold, the maximum voxel intensity for the ray is entered as the projected value, otherwise the median (background) level is entered. Fig. 3 shows modified MIP images for the same set of data and viewing direction as Fig. 1 , for three different values of $K$. While the images in Fig. 3 are necessarily very similar to that in Fig. 1 in terms of the bright pixels associated with blood vessels, they show much less variation in the background. The contrast-to-noise ratio for the modified MIP images is therefore better than for the corresponding MIP. It has to be acknowledged, of course, that the improvement is as a result of lowering the background noise level without any improvement in the estimates of the object pixels. The result is relatively insensitive to the $K$ value chosen.

\section{3D SEGMENTATION}

In order to provide improved time-resolved MRA image sequences of the blood vessels, our reconstruction algorithms [8], [9], [10] require an accurate segmentation of the relatively noisy 3D data set into vessel and background. The method described in Section I produces a segmentation with many false detections because of the effect of overlying structures and the relatively noisy background in MIP images. We propose therefore to use the same principle as employed for the modified MIP in Section II to achieve better 3D segmentation. 

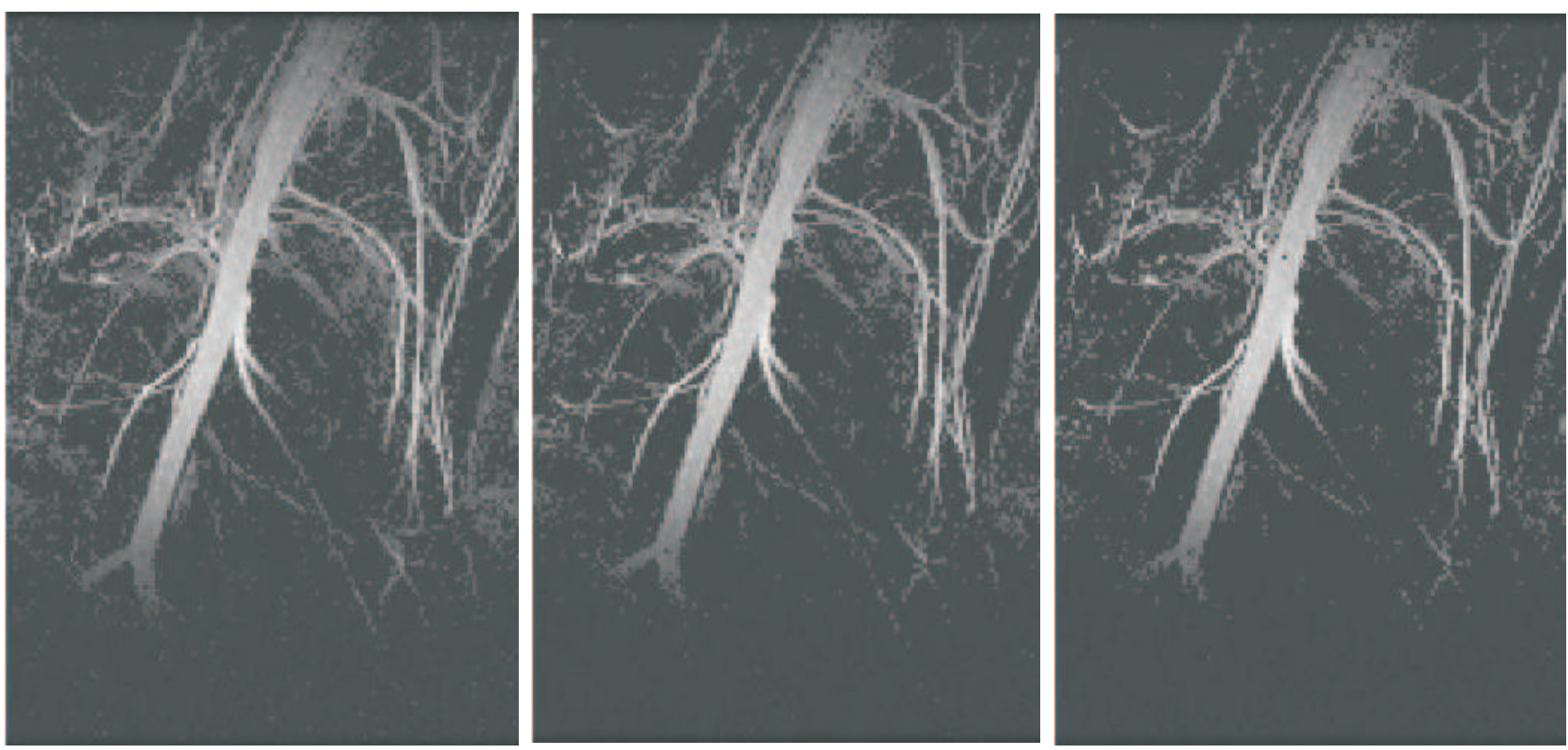

Fig. 3. Coronal modified MIP images formed from the same set of contrast-enhanced MRA data obtained as shown in Fig. $1:($ left $) K=4.5$, $($ centre $) K=$ 5.5, and (right) $K=6.5$.

In the proposed method, for each voxel in the 3D data the histograms of the 3 orthogonal rays in the directions of the Cartesian axes which intersect with that voxel are evaluated. A threshold is calculated for each of the three rays in the same way as described in Section II. Thus for the voxel three thresholds $T_{x}, T_{y}$ and $T_{z}$ are found. If the voxel intensity exceeds at least two of the thresholds (i.e., a majority) the voxel is classed as 'vessel', otherwise it is classed as 'background'.

Comparison of results shows that the best value to use for $K$ in computing $T_{x}, T_{y}$ and $T_{z}$ is in the range 5 to 7.

\section{Results EVALUATion}

To facilitate a quantitative comparison for the modified MIP visualisation method, we used the contrast-to-noise ratio (CNR) which was calculated as follows:

$$
\mathrm{CNR}=\frac{\left(\mu_{V}-\mu_{B}\right)\left(N_{V}+N_{B}\right)^{\frac{1}{2}}}{\left(N_{V} \sigma_{V}^{2}+N_{B} \sigma_{B}^{2}\right)^{\frac{1}{2}}},
$$

where $\mu_{V}$ and $\sigma_{V}^{2}$ are the mean and variance respectively of pixels lying within a vessel, $\mu_{B}$ and $\sigma_{B}^{2}$ are the mean and variance respectively of pixels lying within the background, $N_{V}$ is the number of vessel pixels considered and $N_{B}$ is the number of background pixels considered [13].

In order to check that comparable results were achieved from a range of data sets, we applied the modified MIP method to three data sets using $K=7$. The data sets were each of the knee region and made with similar injections of contrast agent, but obtained from different subjects and with different MRI sequences. In each case those pixels for which voxels in the corresponding ray exceeded the threshold were counted as 'vessel', while the remainder were counted as 'background'. The resulting CNR values for the three data sets were: 5.2971,
6.7756 and 4.5528 . Figs. 4 and 5 show images formed for the second and third of these data sets, both by the standard MIP method (left) and by the modified MIP method (right).

CNR values were also calculated with respect to specific features in the images. For example, for the coronal projection images shown in Figs. 1 and 3, patches were manually selected within the large central blood vessel and one of the small vessels. A larger region was chosen within the part of the images clearly separated from the vessels. The patches chosen had sizes 66 pixels (large vessel), 19 pixels (small vessel) and 2296 pixels (background). The results are shown in Table 1.
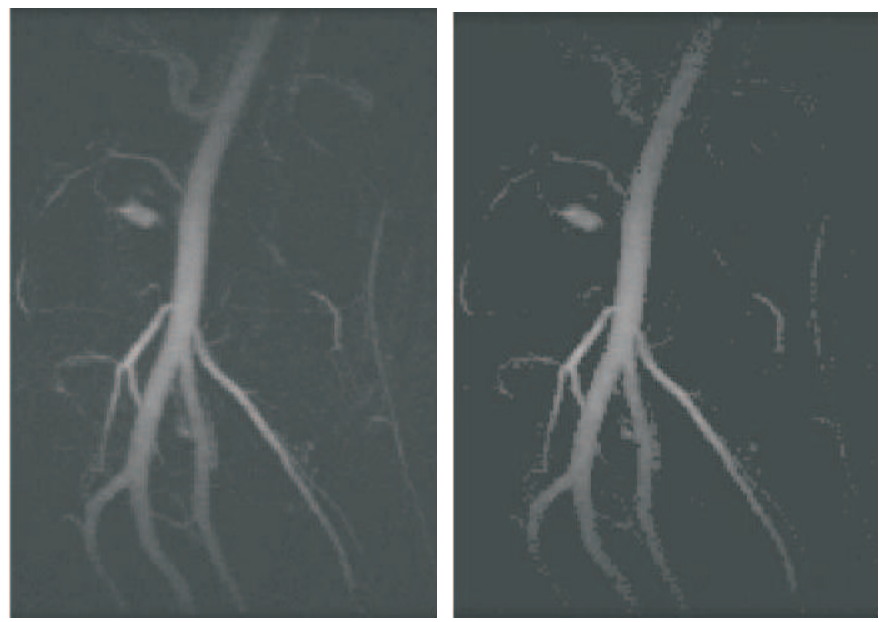

Fig. 4. Projection images for a second 3D data set of the knee region of a healthy volunteer obtained by contrast-enhanced MRA: (left) coronal MIP; and (right) coronal modified MIP $(K=7)$.

To evaluate the success of using the new method for 3D segmentation, we present results for a single coronal slice in Fig. 6 and a single axial slice in Fig. 7. In each figure the left 

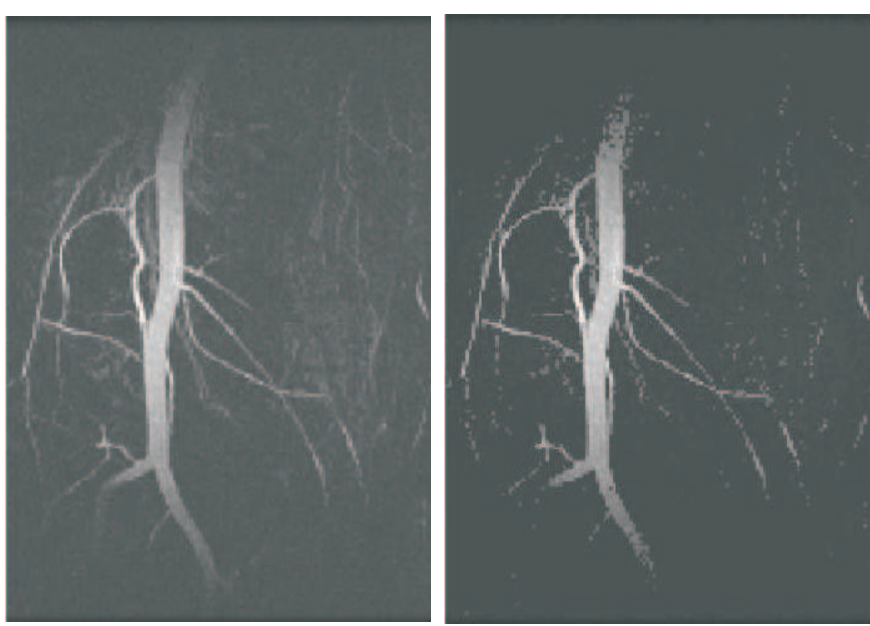

Fig. 5. Projection images for a third 3D data set of the knee region of a healthy volunteer obtained by contrast-enhanced MRA: (left) coronal MIP; and (right) coronal modified MIP $(K=7)$.

\begin{tabular}{c|c|c}
\hline & Standard MIP & Modified MIP \\
\hline \hline large vessel & 43.45 & 84.22 \\
\hline small vessel & 37.88 & 71.43 \\
\hline
\end{tabular}

TABLE I

CNR VALUES BASED ON PATCHES CHOSEN FROM THE CORONAL PROJECTION IMAGES SHOWN IN FIGS. 1 AND 3.

image shows the raw data for the 3D reconstruction (the same data set as used for Figs. 1 and 3, but a single slice only). The centre image shows the crude segmentation generated for that slice by our original MIP-based segmentation [10]. The morphological dilation operations used in forming the ROS have clearly made the ROS a poor fit to the vessels present in the slice. The right image shows the result of performing our modified segmentation with those voxels segmented as background shown black. It is clear that the algorithm successfully forms a tight support region around all sizeable vessels.
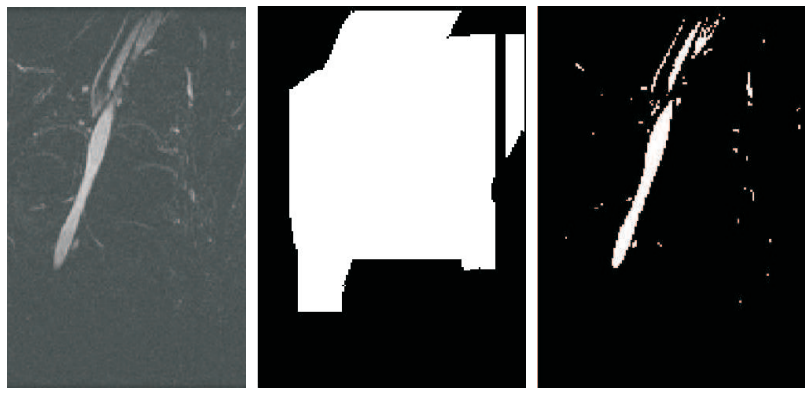

Fig. 6. 3D segmentation using the modified MIP method. A single coronal slice from the same 3D data set depicted in Fig. 1 is shown: (left) raw slice data; (centre) slice segmented by the crude thresholding method; and (right) slice segmented into vessel and non-vessel by the modified MIP method.

\section{Discussion}

Despite the simplicity and widespread acceptance of the maximum intensity projection (MIP) technique for displaying three-dimensional (3D) magnetic resonance angiographic data,
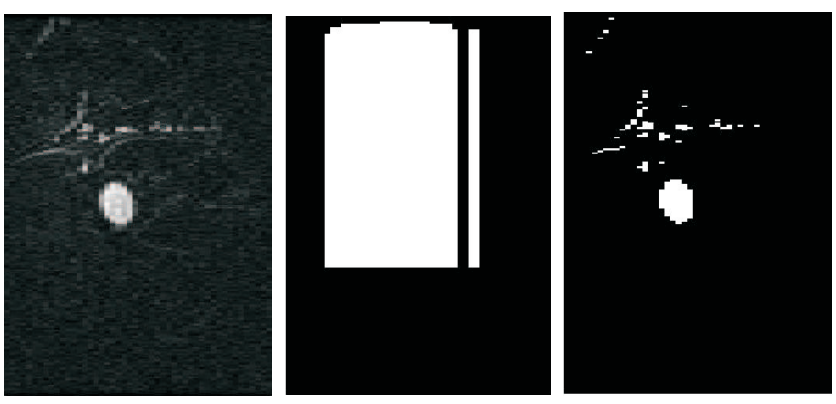

Fig. 7. 3D segmentation using the modified MIP method. A single axial slice from the same 3D data set depicted in Fig. 1 is shown: (left) raw slice data; (centre) slice segmented by the crude thresholding method; and (right) slice segmented into vessel and non-vessel by the modified MIP method.

several disadvantages are associated with MIP. These include an elevated noise level in the background and low contrast between small vessels and background tissue. We have developed a method to complement the conventional MIP and alleviate these problems. Significantly better CNR values are obtained with the modified MIP method according to our preliminary studies. The incorporation of a single parameter allows the new method to be tailored to specific viewing tasks.

The main motivation in developing the modified MIP method was in seeking a way of segmenting 3D MRA data sets. Simple global thresholding of MIP images allowed only fairly gross approximations to the vascular region to be formed. By assessing each voxel on the basis of the three orthogonal rays passing through it and forming thresholds based on robust statistics, it is possible to better discriminate between voxels likely to lie within vessels and those likely to lie outside. The use of the majority operator reduces the possibility that an overlaying structure can influence the segmentation decision.

The ROS formed by the new method (cf. right hand images in Figs. 6 and 7) is 'tight'. Best use of the ROS for MRA reconstruction algorithms requires a somewhat looser support, so we plan to use some form of $3 \mathrm{D}$ dilation operation following the segmentation.

The work presented here is preliminary. Next steps include performing more extensive studies and incorporating the new 3D segmentation method into our MRA reconstruction work [10].

\section{REFERENCES}

[1] S. Roth, "Ray casting for modeling solids," Computer Graphics and Image Processing, vol. 18, pp. 109-144, 1982.

[2] S. Rossnick, G. Laub, et al, "Three dimensional display of blood vessels in MRI," Computers in Cardiology, October 7-10, 1986, Boston, Massachusetts USA, p. 193.

[3] G. Laub, "Displays for MR angiography, "Magnetic Resonance in Medicine, vol. 14(2), p. 222, 1990.

[4] Y. Sun, and D. Parker, "Small vessel enhancement in MRA images using local maximum mean processing," IEEE Transactions on Image Processing, vol. 10, pp. 1687-1699, 2001.

[5] B. Chapman, J. Stapelton, et al, "Intracranial vessel segmentation from time-of-flight MRA using pre-processing of the MIP Z-buffer: accuracy of the ZBS algorithm," Medical Image Analysis, vol. 8, pp. 113-126, 2004.

[6] D. Brown, and S. Riederer, "Contrast-to-noise ratios in maximum intensity projection images," Magnetic Resonance in Medicine, vol. 23, pp. 130-137, 1992. 
[7] Y. Sun, and D. Parker, "Performance analysis of maximum intensity projection algorithm for display of MRA images," IEEE Transactions on Medical Imaging, vol. 18, pp. 1154-1169, 1999.

[8] P.J. Bones, N. Alwesh, T.J. Connolly, and N.D. Blakeley, "Recovery of limited-extent images aliased because of spectral undersampling," JOSA A, vol. 18, pp. 2079-2088, 2001.

[9] B. Wu, R.P. Millane, R. Watts, and P.J. Bones, 'Improved matrix inversion in image plane parallel MRI," Magnetic Resonance Imaging, vol. 27, pp. 942-953, 2009.

[10] B. Wu, "Exploiting Data Sparsity in Parallel Magnetic Resonance Imaging," $\mathrm{PhD}$ thesis (submitted), University of Canterbury, 2009.

[11] N. Aggarwal, Q. Zhao, and Y. Bresler, "Spatio-temporal modeling and minimum redundancy adaptive acquisition in dynamic MRI," Proceedings IEEE Int. Symp. Biomedical Imaging, pp. 737-740, 2002.

[12] R.A. Maronna, R.D. Martin, and V.J. Yohai, "Robust statistics: theory and methods", Wiley, 2006.

[13] D. Brown, and S. Riederer, "Contrast-to-noise ratios in maximum intensity projection images," Magnetic Resonance in Medicine, vol. 23, pp. 130-7, 1992. 